\title{
A Review of Current Clinical Applications of Three- Dimensional Printing in Spine Surgery
}

\author{
Woojin Cho ${ }^{1}$, Alan Varkey Job ${ }^{1}$, Jing Chen ${ }^{1}$, Jung Hwan Baek ${ }^{2}$ \\ ${ }^{1}$ Department of Orthopaedic Surgery, Albert Einstein College of Medicine/Montefiore Medical Center, Bronx, NY, USA \\ ${ }^{2}$ Department of Plastic Surgery, H Plastic Surgery Clinic, Seoul, Korea
}

\begin{abstract}
Three-dimensional (3D) printing is a transformative technology with a potentially wide range of applications in the field of orthopaedic spine surgery. This article aims to review the current applications, limitations, and future developments of 3D printing technology in orthopaedic spine surgery. Current preoperative applications of 3D printing include construction of complex 3D anatomic models for improved visual understanding, preoperative surgical planning, and surgical simulations for resident education. Intraoperatively, 3D printers have been successfully used in surgical guidance systems and in the creation of patient specific implantable devices. Furthermore, 3D printing is revolutionizing the field of regenerative medicine and tissue engineering, allowing construction of biocompatible scaffolds suitable for cell growth and vasculature. Advances in printing technology and evidence of positive clinical outcomes are needed before there is an expansion of 3D printing applied to the clinical setting.
\end{abstract}

Keywords: Three-demensional printing; Spine; Guidance; Templates; Implants

\section{Introduction}

Three-dimensional (3D) printing is a transformative technology that has the potential to greatly impact the field of orthopaedic spine surgery. This review article aims to describe the current technology, clinical applications, future direction, and limitations of 3D printing in spine surgery. Three-D printing has been used in surgical planning for complex spine surgeries since the 1990s [1]. Currently, the technologies role has expanded to both the preoperative and intraoperative setting. Preoperatively, 3D printing is being used to create anatomical accurate models of spinal deformities for resident and patient education. In addition these models are being used for preoperative surgical planning and simulation for complex spinal pathologies. A tactile 3D visual representation provides a better depiction of anatomy than can be achieved through computed tomography (CT)/magnetic resonance imaging (MRI) imaging. Intraoperatively, 3D printing has been used to create surgical guidance systems, templates, and customized patient specific implants. Despite the excellent advantages of 3D printing, such as improved patient outcomes and decreased radiation exposure to patients, 3D printing has faced challenges preventing its widespread use in clinical practice. The field of 3D printing is an important technology that has the potential to transform the practice of orthopaedic spine surgery in the near future.

Received Jul 12, 2017; Revised Aug 7, 2017; Accepted Aug 8, 2017

Corresponding author: Woojin Cho

Albert Einstein College of Medicine/Montefiore Medical Center, 3400 Bainbridge Ave, 6th Floor, Bronx, NY 10467-2404, USA

Tel: +1-718-920-2060, Fax: +1-718-653-1587, E-mail: wcho@montefiore.org 


\section{Printing Techniques and Tissue Engineering Applications}

Additive manufacturing, commonly known as 3D printing or rapid prototyping, utilizes $3 \mathrm{D}$ computer representations to create physical models. The anatomic models begin as two-dimensional images and vectors from CT/ MRI image data. They are converted to $3 \mathrm{D}$ models using specialized $3 \mathrm{D} /$ multiplanar modeling software. Using the digital image, specialized printers build a 3D object by printing thin cross-sections in a layer-by-layer fashion. The earliest version of $3 \mathrm{D}$ printing is known as stereolithography (SLA), patented by Charles Hulk in the 1980s, which utilized ultraviolet light and photocurable resin [2]. Since then, 3D printing technologies have been enhanced and novel processes were developed for a variety of applications from surgical simulations to personalized surgery and tissue engineering.

There exists great diversity among 3D printing technology. Three-D printing is a printing technique that builds 3D model from cross-sectional layers. Three-D printing has been shown to be advantageous in fabricating scaffold with microscopic internal channels and high porosity as the surrounding powders take on the role of supporting material [3]. Three-D printing allowed for the generation of phosphate and strontium-containing scaffolds which stimulated blood vessel formation and osteoblast proliferation $[4,5]$. Several studies utilized $3 \mathrm{D}$ printing to generate hydroxyapatite scaffolds that supports bone tissue development [6,7]. Seitz et al. [8] has fabricated porous ceramic scaffolds from hydroxyapatite powder that is suitable for cell growth and potential bone implantation. Qian et al. [9] further demonstrated the feasibility of 3D printing to construct functionally graded implants by fabricating and characterizing titanium/hydroxyapatite composite.

Fused deposition modeling (FDM) is another printing technique that deposits molten thermoplastic materials into cross sectional layers [3]. Recent advances have allowed creation of scaffold with biocompatible building material. Studies conducted with chondrocytes and osteoblast supports FDM-generated scaffolds in bone tissue engineering [3]. Hydroxyapatite containing scaffolds produced by $3 \mathrm{D}$ printing are also possible with FDM. In combination with amphiphilic polymer, hydroxyapatite scaffolds were generated with favorable osteoconductive properties [10].

SLA is another $3 \mathrm{D}$ printing technology using photocur- able materials capable of photo-crosslinking. The disadvantage of SLA is the scarcity of biocompatible material that also possesses photo-inducible properties. Recent advancement includes the expansion of libraries for such materials [3]. Other more recently developed 3D printing techniques includes selective laser sintering and 3D plotting, all of which have shown great potential in tissue reconstruction, especially in the field of bone tissue engineering [11-13].

Technical limitations to $3 \mathrm{D}$ printing technology must also be further developed. One limiting factor is that the support martial used to fill air spaces will remain inside the cavities due to current inability to remove the material. While there are many successes in generating models of bone and implants, soft tissue, such as nerves and vessels, still need further development. In addition, material texture could improve to resemble the modeled tissue for better simulation.

\section{Accuracy of Three-Dimensional Printing}

Exceptional accuracy and precision of 3D models representing true anatomic structures is critical for $3 \mathrm{D}$ printing to have clinical utility in spine surgery. Studies have validated the potential for 3D-printed models to accurately represent the complex anatomical features of the spine. Wu et al. [14] compared CT images of healthy cervical, thoracic, and lumbar vertebrae against their respective $3 \mathrm{D}$-printed models and found the $3 \mathrm{D}$ models had strong anatomic correlation. Another study assessed the accuracy of 3D-printed models representing cadaveric pelvises and observed no significant difference between them [15]. McMenamin et al. [16] found that 3D-printed models not only accurately represented structural radiologic data but also accurately represented air and fluid filled negative spaces. Modern printing techniques allow for creation of models with high calcium content to accurately mimic the true density of bone [17].

\section{Preoperative Planning Applications}

An advantage provided by $3 \mathrm{D}$-printed spine models is the realistic surgical simulation in preoperative planning, which can be advantageous in preparing for complex spinal pathologies. Xiao et al. [18] was able to successfully preform complex en bloc resections of primary cervical tumors using 3D-printed spine models for preparation 
and visual reference. In another case, 3D models were used in complex craniovertebral surgeries to study inclination of the joints, false articulations, pedicles sizes, and vertebral artery courses preoperatively. Data from the models allowed for preoperative calculation of screw and plate sizes and angle of screw insertion [19]. In the correction of spinal deformity in children with meningomyelocele, personalized 3D printing has been used to create surgical planning models and patient specific spinal instrumentations [20]. Preoperative 3D modeling for surgical planning was used in surgery of a thoracolumbar fracture with dislocation by providing modeling of the sagittal curves and navigation templates for pedicle screws. The procedure had advantages of shorter operation time, less intraoperative blood loss, better recovery of thoracolumbar dislocation, and better Frankel classification when completed with a 3D model [21]. Rapid prototyping of 3D models have been used in surgical planning for revision lumbar discectomy, resulting in reduced operation time and perioperative blood loss [22]. A study on posterior corrective surgery for Lenke 1 adolescent idiopathic scoliosis (AIS) using preoperative planning with 3D-printed spine models found significantly shorter operation time, significantly less blood loss and transfusion volume, and higher postoperative hemoglobin compared to the same surgery without the 3D model. However, no significant differences were observed in complication rate, length of hospital stay, postoperative radiological outcomes, or pedicle screw misalignments between the two groups [23].

Some limitations exist in the application of 3D printing in the preoperative setting. Although studies do show multiple advantages in the use of 3D-printed models in preoperative planning, in many cases, such as in Lenke 1 AIS, this extra step is often unnecessary. Although the models may provide shorter operation time, one must also consider the additional time required to design and construct the model preoperatively. Lastly, due to the time delay of designing and constructing 3D models with the current technology, the technology currently has limited use in the orthopaedic trauma setting. However, there have been reported cases of its use in preoperative planning for acetabular reconstruction following trauma [24]. Advancements in the speed and availability of rapid prototyping technology may allow for more widespread use in the trauma setting. Therefore, authors think that 3D printer will be more useful in more complex cases.

\section{Trainee and Patients Education Applications}

Prior to the development of 3D printing, cadaveric models were traditionally used for educational purposes and surgical simulation. However, they are limited in their usage due to shortage of donations, and health and safety issues [14]. A variety of factors, such as work hour restrictions and challenges from complex cases may reduce surgical confidence in residents. 3D-printed spine models serve as an alternative to cadavers and have increased capacity for customizability in educational purposes. The 3D-printed models have a promising future in providing state-of-theart training simulations of surgical experiences for residents and even practitioners [25].

Hughes et al. [26] reported usefulness of 3D models in navigating complex deformities and improving anatomical understanding for training. In a study using 3D models of cervical spines and 3D-printed prototyping drilling templates, residents demonstrated the ability to successfully and precisely accomplish proper trough positioning on cadavers for expansive open door laminoplasties, a critical and technically demanding step of the procedure [27]. Printed spine models with characteristics mimicking true bone density have been used to help trainees become competent in needle placement for image-guided diagnostic and therapeutic spinal procedures $[17,28]$.

Lastly, 3D-printed spine models have also been reported to be successfully applied in the informed consent process. Patient specific 3D-printed spine models have helped guide patients in the decision making process by educating them on their spinal pathologies and the surgical procedures $[29,30]$.

\section{Intraoperative Applications: Guidance Systems}

A primary clinical application of 3D printing in spinal surgery is the creation of drill guidance plates for the insertion of pedicle screws. Using CT thin-slice data of the patient's spine, computer software's design navigation templates for pedicle screw fixation that is $3 \mathrm{D}$-printed preoperatively and then sterilized for intraoperative use $[31,32]$. Cervical pedicle screw (CPS) fixation is excellent for posterior cervical fixation due to its biomechanical advantages. However, the narrow clearance for the CPS increases risk for neural and vascular injuries; the 3D-printed template guided procedure is a solution for 
reducing the risk. In a cadaveric study, patient-specific drill guide templates used for cervical pedicle fixation improved accuracy of pedicle screw placement [33]. This template guided method for pedicle screw insertion has also been applied to in vivo cases at all vertebral levels. Regarding CPS fixation, multiple studies comparing pedicle screw insertion via $3 \mathrm{D}$ template versus fluoroscopy have shown improved accuracy of placement, improved precision, and reduced complications in the $3 \mathrm{D}$ template guided method. Authors also agree the reduced operative time, ease-of-use, moderate cost, and ability to insert cervical screws without radiation are additional benefits $[31,32,34]$. Three-D template guided placement of pedicle screws have also been conducted on thoracic vertebrae with equally impressive accuracy, safety, and convenience $[35,36]$. A study of lumbar pedicle screw fixation by Chen et al. [37], found no significant difference in the accuracy of 3D template guided and fluoroscopic screw insertion, with $100 \%$ and $98.4 \%$ accurate placement respectively. However, the study did find significantly reduced operative time, blood loss, and radiation dose in the $3 \mathrm{D}$ template guided group [37]. Lastly, studies using 3D template guides for multilevel pedicle screw fixations also found improved accuracy of placement, reduced operative time, reduced radiation exposure, and reduced incidence of cortical perforation, all while remaining an economical approach [38,39].

There are still multiple limitations preventing the widespread use of 3D printing in the intraoperative setting. Utilizing 3D technology often requires a specialist with background in managing 3D software to perform segmentation of a 3D model for printing. In addition, processing $3 \mathrm{D}$ imaging data, designing, and printing intraoperative guidance systems can be a time intensive process which has limited its widespread use in hospitals. Use of 3D printing techniques adds an additional cost to the surgery. However, recent advances have allowed for all steps, from generating digital 3D models to segmentation and printing, to be performed on a single computer in a user-friendly manner. The process is becoming more streamlined with advancements in software and automation. Although imaging processing and printing can take several hours prior to the surgery, the guidance systems have been shown to reduce intraoperative times. One review of 3D spine systems in surgery concluded that 10 minutes saved in the operating room is equivalent in monetary terms to one hour spent in preparation of the guidance system [40].

\section{Intraoperative Applications: Implants}

Three-D printing encourages the advancement of personalized surgery. Commercially-available implants will not always match patient specific anatomy but 3D printing technology allows for the development of customizable surgical tools and implants. Xu et al. [41] fabricated a 3D titanium alloy axial vertebral body that was implanted for upper cervical spinal reconstruction following a C2 Ewing sarcoma resection. Tumor resection is particularly challenging in the atlantoaxial region due to complexity of the anatomy, necessity for extensive resection, and a lack of specialized implants for reconstruction [41]. Kim et al. [42] successfully developed a patient specific custom 3D-printed implant for use in pelvic reconstruction following hemisacral resection. Multiple cases have reported successful use of patient specific 3D-printed implants for spinal fusion in complex spinal pathologies, including posterior fixation implants, and anterior and posterior intervertebral fusion cage implants [43-46]. The implants provided improved load bearing surface, lowered the rate of implant dislocation and subsidence, provided excellent primary stabilization, shortened time of procedure, improved correction of deformity, decreased blood loss, and reduced risk of neurovascular compromise $[20,40,46]$. Advances in tissue regeneration and printing techniques may allow for 3D printing of intervertebral discs in the future $[47,48]$. Although the technology enabling accurate fabrication of patient-specific spinal implants is available, literature on its utilization is currently rare.

There are limitations to the use of 3D-printed implants. As $3 \mathrm{D}$ printing for clinical use is a recent development, current studies on 3D implants lack long term follow-up data of post-surgical clinical outcomes. Data supporting positive clinical outcomes are needed to justify the added expense of 3D printing in spine surgery. For the safety of the patients, Food and Drug Administration approval is critical before inserting 3D-printed implants into the patients' body.

\section{Conclusions}

Three-D printing technology in spine surgery is currently being used for preoperative planning, trainee and patient education, intraoperative guidance systems, intraopera- 
tive implants, and is revolutionizing the field of tissue engineering. Advances in printing technology and evidence of positive clinical outcomes are needed before there is an expansion of $3 \mathrm{D}$ printing applied to the clinical setting.

\section{Conflict of Interest}

No potential conflict of interest relevant to this article was reported.

\section{Acknowledgments}

Institutional Review Board approval is not applicable. The authors give permission to reproduce copyrighted materials or signed patient consent forms.

\section{References}

1. Grant CA, Izatt MT, Labrom RD, Askin GN, Glatt V. Use of $3 \mathrm{D}$ printing in complex spinal surgery: historical perspectives, current usage, and future directions. Tech Orthop 2016;31:172-80.

2. Zhang X, Zhang Y. Tissue engineering applications of three-dimensional bioprinting. Cell Biochem Biophys 2015;72:777-82.

3. Chia $\mathrm{HN}, \mathrm{Wu} \mathrm{BM}$. Recent advances in 3D printing of biomaterials. J Biol Eng 2015;9:4.

4. Zhang J, Zhao S, Zhu Y, et al. Three-dimensional printing of strontium-containing mesoporous bioactive glass scaffolds for bone regeneration. Acta Biomater 2014;10:2269-81.

5. Tarafder S, Davies NM, Bandyopadhyay A, Bose S. $3 \mathrm{D}$ printed tricalcium phosphate scaffolds: effect of $\mathrm{SrO}$ and $\mathrm{MgO}$ doping on in vivo osteogenesis in a rat distal femoral defect model. Biomater Sci 2013;1:1250-9.

6. Simon JL, Rekow ED, Thompson VP, Beam H, Ricci JL, Parsons JR. MicroCT analysis of hydroxyapatite bone repair scaffolds created via three-dimensional printing for evaluating the effects of scaffold architecture on bone ingrowth. J Biomed Mater Res A 2008;85:371-7.

7. Wang Y, Li X, Wei Q, Yang M, Wei S. Study on the mechanical properties of three-dimensional directly binding hydroxyapatite powder. Cell Biochem Biophys 2015;72:289-95.

8. Seitz H, Rieder W, Irsen S, Leukers B, Tille C. Three- dimensional printing of porous ceramic scaffolds for bone tissue engineering. J Biomed Mater Res B Appl Biomater 2005;74:782-8.

9. Qian C, Zhang F, Sun J. Fabrication of Ti/HA composite and functionally graded implant by three-dimensional printing. Biomed Mater Eng 2015;25:12736.

10. Kutikov AB, Gurijala A, Song J. Rapid prototyping amphiphilic polymer/hydroxyapatite composite scaffolds with hydration-induced self-fixation behavior. Tissue Eng Part C Methods 2015;21:229-41.

11. Kim K, Yeatts A, Dean D, Fisher JP. Stereolithographic bone scaffold design parameters: osteogenic differentiation and signal expression. Tissue Eng Part B Rev 2010;16:523-39.

12. Van Bael S, Desmet T, Chai YC, et al. In vitro cellbiological performance and structural characterization of selective laser sintered and plasma surface functionalized polycaprolactone scaffolds for bone regeneration. Mater Sci Eng C Mater Biol Appl 2013;33:3404-12.

13. Williams JM, Adewunmi A, Schek RM, et al. Bone tissue engineering using polycaprolactone scaffolds fabricated via selective laser sintering. Biomaterials 2005;26:4817-27.

14. Wu AM, Shao ZX, Wang JS, et al. The accuracy of a method for printing three-dimensional spinal models. PLoS One 2015;10:e0124291.

15. Wu XB, Wang JQ, Zhao CP, et al. Printed threedimensional anatomic templates for virtual preoperative planning before reconstruction of old pelvic injuries: initial results. Chin Med J (Engl) 2015;128:47782.

16. McMenamin PG, Quayle MR, McHenry CR, Adams JW. The production of anatomical teaching resources using three-dimensional (3D) printing technology. Anat Sci Educ 2014;7:479-86.

17. West SJ, Mari JM, Khan A, et al. Development of an ultrasound phantom for spinal injections with 3-dimensional printing. Reg Anesth Pain Med 2014;39:429-33.

18. Xiao JR, Huang WD, Yang XH, et al. En bloc resection of primary malignant bone tumor in the cervical spine based on 3-dimensional printing technology. Orthop Surg 2016;8:171-8.

19. Goel A, Jankharia B, Shah A, Sathe P. Three-dimensional models: an emerging investigational revolution 
for craniovertebral junction surgery. J Neurosurg Spine 2016;25:740-4.

20. Karlin L, Weinstock P, Hedequist D, Prabhu SP. The surgical treatment of spinal deformity in children with myelomeningocele: the role of personalized three-dimensional printed models. J Pediatr Orthop B 2017;26:375-82.

21. Wu C, Tan L, Lin X, Hu H. Clinical application of individualized reference model of sagittal curves and navigation templates of pedicle screw by threedimensional printing technique for thoracolumbar fracture with dislocation. Zhongguo Xiu Fu Chong Jian Wai Ke Za Zhi 2015;29:1381-8.

22. Li C, Yang M, Xie Y, et al. Application of the polystyrene model made by $3-\mathrm{D}$ printing rapid prototyping technology for operation planning in revision lumbar discectomy. J Orthop Sci 2015;20:475-80.

23. Yang M, Li C, Li Y, Zhao Y, et al. Application of 3D rapid prototyping technology in posterior corrective surgery for Lenke 1 adolescent idiopathic scoliosis patients. Medicine (Baltimore) 2015;94:e582.

24. Duncan JM, Nahas S, Akhtar K, Daurka J. The use of a $3 \mathrm{D}$ printer in pre-operative planning for a patient requiring acetabular reconstructive surgery. J Orthop Case Rep 2015;5:23-5.

25. Rehder R, Abd-El-Barr M, Hooten K, Weinstock P, Madsen JR, Cohen AR. The role of simulation in neurosurgery. Childs Nerv Syst 2016;32:43-54.

26. Hughes A, Soden P, Abdulkarim A, McMahon C, Hurson $C$. The use of rapid prototyping and $3 \mathrm{D}$ printing in revision hip arthroplasty. Bone Joint J 2014;96B(SUPP 10):2.

27. Rong $\mathrm{X}$, Wang $\mathrm{B}$, Chen $\mathrm{H}$, et al. Use of rapid prototyping drill template for the expansive open door laminoplasty: a cadaveric study. Clin Neurol Neurosurg 2016;150:13-7.

28. Javan R, Bansal M, Tangestanipoor A. A prototype hybrid gypsum-based 3-dimensional printed training model for computed tomography-guided spinal pain management. J Comput Assist Tomogr 2016;40:62631.

29. Madrazo I, Zamorano C, Magallon E, et al. Stereolithography in spine pathology: a 2-case report. Surg Neurol 2009;72:272-5; discussion 275.

30. Liew Y, Beveridge E, Demetriades AK, Hughes MA. $3 \mathrm{D}$ printing of patient-specific anatomy: a tool to improve patient consent and enhance imaging inter- pretation by trainees. Br J Neurosurg 2015;29:712-4.

31. Guo F, Dai J, Zhang J, et al. Individualized 3D printing navigation template for pedicle screw fixation in upper cervical spine. PLoS One 2017;12:e0171509.

32. Deng T, Jiang M, Lei Q, Cai L, Chen L. The accuracy and the safety of individualized $3 \mathrm{D}$ printing screws insertion templates for cervical screw insertion. Comput Assist Surg (Abingdon) 2016;21:143-9.

33. Bundoc RC, Delgado GG, Grozman SA. A novel patient-specific drill guide template for pedicle screw insertion into the subaxial cervical spine utilizing stereolithographic modelling: an in vitro study. Asian Spine J 2017;11:4-14.

34. Sugawara T, Higashiyama N, Kaneyama S, Sumi M. Accurate and simple screw insertion procedure with patient-specific screw guide templates for posterior C1-C2 fixation. Spine (Phila Pa 1976) 2017;42:E3406.

35. Takemoto M, Fujibayashi S, Ota E, et al. Additivemanufactured patient-specific titanium templates for thoracic pedicle screw placement: novel design with reduced contact area. Eur Spine J 2016;25:1698-705.

36. Chen H, Guo K, Yang H, Wu D, Yuan F. Thoracic pedicle screw placement guide plate produced by three-dimensional (3-D) laser printing. Med Sci Monit 2016;22:1682-6.

37. Chen H, Wu D, Yang H, Guo K. Clinical use of 3D printing guide plate in posterior lumbar pedicle screw fixation. Med Sci Monit 2015;21:3948-54.

38. Liu K, Zhang Q, Li X, et al. Preliminary application of a multi-level 3D printing drill guide template for pedicle screw placement in severe and rigid scoliosis. Eur Spine J 2017;26:1684-9.

39. Sugawara T, Higashiyama N, Kaneyama S, et al. Multistep pedicle screw insertion procedure with patientspecific lamina fit-and-lock templates for the thoracic spine: clinical article. J Neurosurg Spine 2013;19:18590.

40. Martelli N, Serrano C, van den Brink H, et al. Advantages and disadvantages of 3-dimensional printing in surgery: a systematic review. Surgery 2016;159:1485500.

41. Xu N, Wei F, Liu X, et al. Reconstruction of the upper cervical spine using a personalized 3D-printed vertebral body in an adolescent with ewing sarcoma. Spine (Phila Pa 1976) 2016;41:E50-4.

42. Kim D, Lim JY, Shim KW, et al. Sacral reconstruction 
with a 3D-printed implant after hemisacrectomy in a patient with sacral osteosarcoma: 1-year follow-up result. Yonsei Med J 2017;58:453-7.

43. Serra T, Capelli C, Toumpaniari R, et al. Design and fabrication of 3D-printed anatomically shaped lumbar cage for intervertebral disc (IVD) degeneration treatment. Biofabrication 2016;8:035001.

44. Phan K, Sgro A, Maharaj MM, D'Urso P, Mobbs RJ. Application of a 3D custom printed patient specific spinal implant for C1/2 arthrodesis. J Spine Surg 2016;2:314-8.

45. Mobbs RJ, Coughlan M, Thompson R, Sutterlin CE $3 \mathrm{rd}$, Phan K. The utility of 3D printing for surgical planning and patient-specific implant design for complex spinal pathologies: case report. J Neurosurg Spine 2017;26:513-8.

46. Spetzger U, Frasca M, Konig SA. Surgical planning, manufacturing and implantation of an individualized cervical fusion titanium cage using patient-specific data. Eur Spine J 2016;25:2239-46.

47. Yang Z, Li C, Sun H. Research advances of threedimension printing technology in vertebrae and intervertebral disc tissue engineering. Zhejiang Da Xue Xue Bao Yi Xue Ban 2016;45:141-6.

48. Rosenzweig DH, Carelli E, Steffen T, Jarzem P, Haglund L. 3D-printed ABS and PLA scaffolds for cartilage and nucleus pulposus tissue regeneration. Int $J$ Mol Sci 2015;16:15118-35. 